\title{
COMPACT, SCALABLE, HIGH-RESOLUTION, MEMS-ENABLED TACTILE DISPLAYS
}

\author{
X. Xie ${ }^{1 *}$, Y. Zaitsev ${ }^{I}$, L.F. Velásquez-García ${ }^{2}, S$. Teller $^{2}$, and C. Livermore ${ }^{l}$ \\ ${ }^{1}$ Northeastern University, Boston, Massachusetts, USA \\ ${ }^{2}$ Massachusetts Institute of Technology, Cambridge, Massachusetts, USA
}

\begin{abstract}
The design, fabrication, and test of a new type of tactile display for the blind is reported. An array of piezoelectric extensional actuators vibrates in plane, and microfabricated scissor mechanisms convert the in-plane actuations into robust, higher-amplitude, out-of-plane vibrations. Information can be conveyed by varying the vibration patterns in space and time. Analytical and FEM models were used to design individual tactile elements, which were implemented with PZT actuators and both SU-8 and 3D-printed scissor amplifiers. The measured displacements of $3 \mathrm{~mm} \times 10 \mathrm{~mm}$ tactile elements exceed $10 \mu \mathrm{m}$, in agreement with models, with measured forces exceeding $45 \mathrm{mN}$. The creation of a 28 -element prototype is also reported.
\end{abstract}

\section{INTRODUCTION}

Providing information to those who are blind or have low vision is critical for enhancing mobility, situational awareness, education, and more. Tactile information delivery can be effective, rapid, and private. Examples include Braille [1,2] and the Optacon [3], both of which convey text to the user through the motion of several-centimeter-long piezoelectric bending beam actuators. Graphical information that cannot be expressed as text is most commonly embossed on paper. Although refreshable 2D graphical interfaces are preferred, it is challenging to create actuators that are compact enough to be arrayed into an unlimited number of rows and columns while still being robust, easy to sense, and rapidly switchable. Electroactive polymer actuators are small enough to be arrayed with a few millimeter pitch and provide quasistatic millimeter-scale actuations, but they typically have actuation times on the order of seconds (see [4] and references therein). An alternative integrates piezoelectric bending beam actuators perpendicular to the tactile sensing plane, enabling large bending beam actuators to be tightly packed for fully $2 \mathrm{D}$ displays $[5,6]$. MEMS technology is also increasingly being leveraged to create tactile displays, as in [7-9]. Important challenges nonetheless remain, including spatial resolution, refresh rate, and cost.

Ideally, the display's resolution should leverage the approximately one receptor per $\mathrm{mm}^{2}$ spacing of mechanoreceptors in human finger pads and be extendable to full $2 \mathrm{D}$. It should be refreshable in real time, allowing the contents of the display to keep up with rapidly changing inputs. The display should also code information in a way that is easily detected and interpreted. Since humans are much more sensitive to motions and changing stimuli than they are to static patterns [10], the display should code information not only as static patterns, but also as simulated motion against the user's finger pads. Finally, its power consumption should be compatible with portable use, and it should be manufacturable by efficiently scalable means to ensure that its cost is compatible with the resources of its target user base. Although existing displays have met various subsets of these requirements, no existing display has been able to meet all of these requirements simultaneously.

This paper presents the concept, design and modeling, fabrication, and characterization of a new type of tactile actuator created to target these requirements. This new actuator concept comprises a single-layer, 2D array of in-plane, extensional piezoelectric actuators, each of which is capped with a scissor amplifier and blunt "pin" that the user feels with his or her fingers (Figure 1). When the extensional actuators expand and contract, the tops of the scissors fall and rise. The ratio of scissor amplitude to actuator amplitude depends on the scissor angle. In the final display, each actuator is driven with an oscillating voltage to create patterns of vibration on the display surface.

This display concept is dictated by the requirements above. To minimize cost, the design avoids the large, multi-layer actuators of refreshable Braille. The tactile elements (tactels) comprise a single layer of actuators to enable scalable integration to $2 \mathrm{D}$. The technology is compatible with down-scaling tactels to the $1-2 \mathrm{~mm}^{2}$ scale, but actuators with such a small footprint can be expected to create smaller vibrations than larger actuators can. The actuators are therefore designed to operate between tens and hundreds of $\mathrm{Hz}$, where humans sense small actuations most readily [11]. This high frequency design is also consistent with rapid refreshability and the creation of moving patterns. The use of high frequency actuations eliminates polymer actuators and supports the use of piezoelectric, magnetic, or shape memory alloy actuators. Among these, the need to minimize power favors piezoelectrics. Finally, the actuator plus scissor amplifier architecture maximizes the vibrational amplitudes that the system can create from a small extensional actuator.

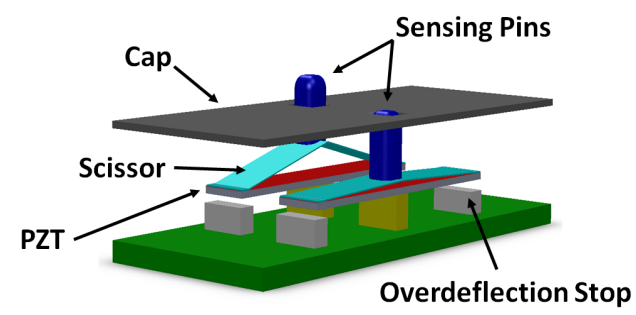

Figure 1: Schematic diagram of piezoelectric extension actuators (red) topped by scissor amplifiers (light blue) and cap plate.

\section{DESIGN}

\section{Device Specifications}

The initial specification for vibrational amplitude was set to $>10 \mu \mathrm{m}$ to accommodate the wide range of human finger sensitivities at tens to hundreds of $\mathrm{Hz}$. This threshold has been validated through laboratory testing of the devices presented below. Although many individuals can detect vibrations at or below the few micron scale, the ability to use amplitudes of $>10$ $\mu \mathrm{m}$ remains useful. In practice, the frequency can be varied between $10 \mathrm{~Hz}$ and $400 \mathrm{~Hz}$ to create different user experiences.

Actuator force can also matter for sensing; if the tactile element has insufficient stiffness, the force that it applies to the user's fingers may be too small for robust detection. A design specification of $>10 \mathrm{mN}$ of oscillatory force for most users is chosen by correlating measured forces with the user experience.

\section{Ideal Tactel Performance}

The piezoelectric extensional actuators comprise y-poled lead zirconate titanate (PZT) bilayer beams that are actuated by 
applying a voltage between electrodes on their top and bottom surfaces. The scissor converts the resulting lengthwise extension or contraction into vertical displacement. For a scissor with ideal pinned hinges, the scissor's vertical displacement $\Delta \mathrm{y}$ is related to the PZT's lengthwise extension $\Delta \mathrm{x}$ by $\Delta y=\Delta x \cot (\theta)$, where $\theta$ is the angle between scissor and actuator (Figure 2). The ratio of vertical displacement to horizontal displacement (the amplification factor) can be large when the scissor angle $\theta$ is small. For example, an angle of $1.25^{\circ}$ corresponds to an amplification factor of $>45$.

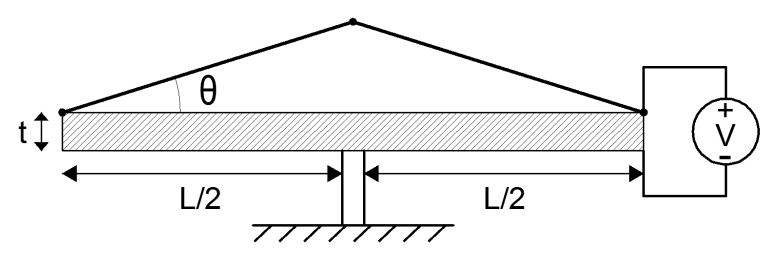

Figure 2: Geometrical parameters for tactel with ideal scissor.

User-applied loads during sensing can impart forces to the PZT beams and scissor amplifiers, affecting the system's performance. For an ideal scissor with rigid arms and pinned hinges, the vertical force $\mathrm{F}$ applied at the scissor's peak produces larger axial forces of $\mathrm{F} /(2 \sin (\theta))$ in the scissor arms. The axial forces in turn apply vertical forces of $F / 2$ and horizontal forces of $\operatorname{Fcot}(\theta) / 2$ at the ends of the PZT beam. The vertical forces tend to bend the PZT and must be limited to prevent breakage. The horizontal forces tend to stretch the PZT and will oppose its contraction. The relationship among the horizontal force from the user, the blocking force, and the target displacement drive the design. A smaller scissor angle offers larger amplification, but a larger scissor angle reduces deflection limits due to the blocking force. Figure 3 illustrates the amplification/blocking force tradeoff by plotting predicted vibrational amplitude vs. scissor angle for tactels using both a MEMS scale actuator $(2500 \mu \mathrm{m} \times 400 \mu \mathrm{m} \times$ $250 \mu \mathrm{m})$ with an applied voltage of $150 \mathrm{~V}$ and a milliscale actuator $(10 \mathrm{~mm} \times 3 \mathrm{~mm} \times 0.38 \mathrm{~mm})$ with an applied voltage of $100 \mathrm{~V}$. The user-applied force per tactel is taken to be $0.1 \mathrm{~N}$. The maximum deflections are calculated as $15.5 \mu \mathrm{m}$ at an angle of $1.1^{\circ}$ and 55.1 $\mu \mathrm{m}$ at $0.25^{\circ}$ for MEMS and milliscale tactels, respectively. The optimal scissor angle for this applied force is therefore in the range of about $1^{\circ}$ or less, depending on the size scale.

Supporting the actuator at the center frees its ends to move in extension and contraction, but the suspended ends can bend or break under applied loads. The maximum stress in the PZT under an applied load of $0.1 \mathrm{~N}$ was calculated at $40 \mathrm{MPa}$ and $26 \mathrm{MPa}$ for the MEMS-scale and milliscale devices, respectively, less than the predicted failure stress of $90 \mathrm{MPa}$. Deflection-limiters are included for additional safety. First, the ends of the actuator beams rest on underlying support surfaces. Second, the tactile display is capped by a perforated plate. The user can sense the pins' vibration where they protrude through the holes, but the cap prevents overdeflection of the tactile elements under load. The cap also provides electrical isolation and prevents tactile distraction.

The key advantage of this architecture as compared with the bending beam actuators of refreshable Braille is that it enables both robustness and large amplitude actuations. The thick, short extensional actuator creates large actuation forces while providing excellent robustness against bending under user-applied load, and the scissor amplifier converts the resulting small, in-plane deflections into large vibrations. In contrast, a piezoelectric bending beam of comparable length would have to be thinner than the actuators used here to achieve sufficient vibrational amplitude, and its thinness would sacrifice robustness against applied forces.

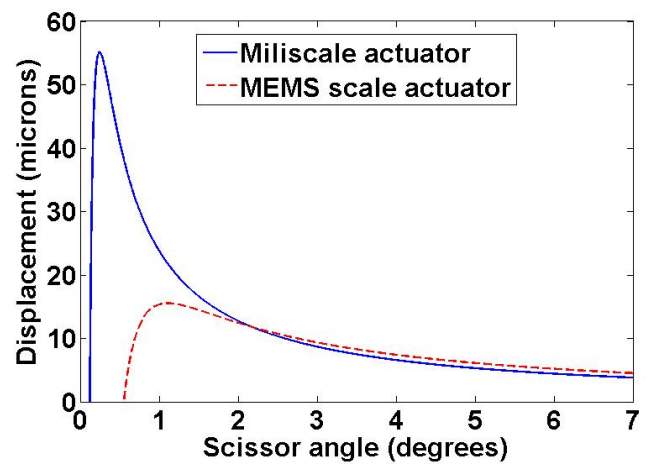

Figure 3: Plot of predicted deflection amplitude as a function of scissor angle for actuators at the MEMS and milli scales.

\section{Scissor Design}

For ease of fabrication, the present scissors use bending flexures in place of pinned hinges. Figure 4 a shows a diagram of the flexural scissor along with its integrated sensing pin. The dimensions of the nominally rigid elements and the flexural hinges are chosen to ensure that the actuator force is sufficient to make the peak of the scissor vibrate and that the scissor does not deform excessively or experience excessive stress under applied loads. Finite element analysis (FEA) was used to predict the performance of the scissors under actuation and user-applied loads. To avoid fatigue, the maximum acceptable stress was set to $100 \mathrm{MPa}$, about 20 times less than the failure stress of SU-8 and the 3D printed material. Based on these results, the hinges are designed with thickness $t_{h}$ of $300 \mu \mathrm{m}$ for the SU-8 hinges and $400 \mu \mathrm{m}$ for the 3D printed hinges; the hinge widths $w_{h}$ are designed as $1.5 \mathrm{~mm}$ and 2.8 $\mathrm{mm}$ for the SU-8 and 3D-printed scissors, respectively. The hinge length $l_{h}$ for both scissor types is $800 \mu \mathrm{m}$ out of a total $9.6 \mathrm{~mm}$ length. Each scissor includes a $6 \mathrm{~mm}$ tall sensing pin. The FEA results of Figure $4 \mathrm{~b}$ show that the predicted peak displacement of an SU-8 scissor with the final, as-fabricated dimensions under an anchor displacement corresponding to an applied voltage of $95 \mathrm{~V}$ is $2 \mu \mathrm{m}$ (corresponding to a peak-to-peak amplitude of $4 \mu \mathrm{m}$ ). The maximum stress in this case is $34 \mathrm{MPa}$.
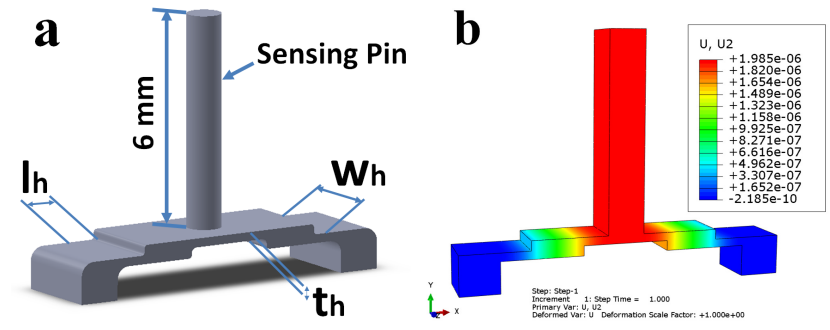

Figure 4: (a) Diagram of scissor with flexural hinges and (b) deformation ( $\mu \mathrm{m}$ ) of SU-8 scissor under $95 \mathrm{~V}$ predicted using FEA.

\section{FABRICATION}

The individual tactels include a combination of micro and milliscale features. Custom y-poled PZT actuators (Piezo Systems, Inc.), laser cut to $10 \mathrm{~mm} \times 3 \mathrm{~mm} \times 380 \mu \mathrm{m}$, comprise the in-plane actuators. The scissor actuators were manufactured by two 
methods, photolithographic definition in $1.5 \mathrm{~mm}$ thick SU-8 and $3 \mathrm{D}$ printing. To pattern the SU-8 scissors, $40 \mathrm{~nm}$ of OmniCoat (MicroChem) was spun onto a silicon substrate in three layers. A $1.5 \mathrm{~mm}$ thick double layer of SU-8 2150 (MicroChem) was spun over the OmniCoat and patterned photolithographically. The Omnicoat was dissolved to lift the SU-8 scissors off the substrate. The 3D-printed scissors were defined from Veroblack Fullcure 870 UV-curable resin in an Objet Eden 333 3D printer. The as-fabricated hinge dimensions are $800 \mu \mathrm{m}$ long x $1470 \mu \mathrm{m}$ wide $\mathrm{x}$ $270 \mu \mathrm{m}$ thick and $800 \mu \mathrm{m}$ long x $2900 \mu \mathrm{m}$ wide x $390 \mu \mathrm{m}$ thick for the SU-8 and 3D-printed scissors, respectively. The difference in hinge thickness primarily reflects the use of two different designs. The difference in scissor width reflects SU-8 process constraints.

Although larger arrays of tactels would ultimately be soldered to a base plate (e.g. a printed circuit board), the devices for single tactel tests and small array tests were connected by soldering wires to nickel electrodes on the upper and lower actuator surfaces. The scissors were adhered to the actuators with a cyanoacrylate adhesive. For single tactel testing, the alignment of the scissor to the actuator did not affect performance, and no provisions were made for alignment. The final tactels are shown in Figure 5.

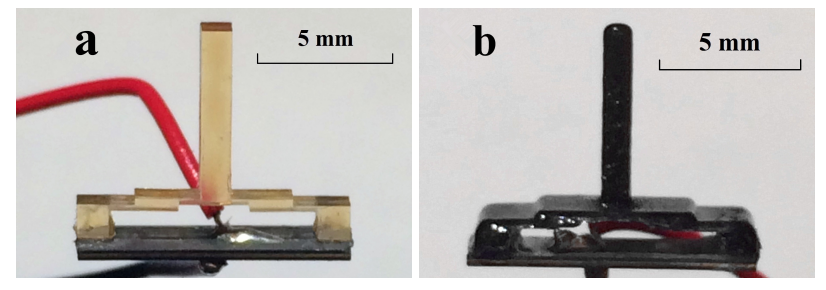

Figure 5: Photographs of fabricated tactels with (a) SU-8 and (b) $3 D$ printed scissors.

For array fabrication, alignment is critical because the sensing pins must align with the holes in the cap plate. The scissors were mechanically aligned to the actuators using a reference surface. Twenty-eight actuators and scissors were then aligned by electrically insulating mechanical stops in a passive alignment plate. The tactels form an offset array to minimize pitch. A thin foam interlayer placed between the actuators and the alignment plate prevents parasitic vibrations on neighboring pins but also somewhat reduces the vibrational signal at the pin. The electrical wires pass through vias in the alignment plate and are soldered to an underlying printed circuit board. The alignment plate provides mechanical support to prevent deflection of the actuator under load. A mechanical spacer controls the height of the cap plate to ensure that the pins protrude by approximately $300 \mu \mathrm{m}$, and all plates are aligned relative to each other by a set of alignment pins. The package and its alignment features are shown in Figure 6.

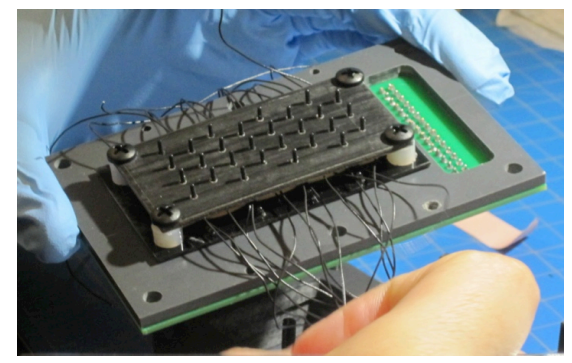

Figure 6: Photograph of 28-element tactile array during assembly.

\section{EXPERIMENT}

The individual tactels were mounted on a testing stage under an optical stereomicroscope so that deformations of the scissor were visible in the microscope's viewing plane, and displacement was measured under actuation. Square wave voltages with a frequency of $0.5 \mathrm{~Hz}$ were output by a function generator and amplified to achieve peak voltage amplitudes of up to $170 \mathrm{~V}$. The voltage was increased in $10 \mathrm{~V}$ steps from a starting value of $40 \mathrm{~V}$ to the maximum value of $170 \mathrm{~V}$. Two or three images of the tactel in each of its maximum and minimum positions for each voltage were captured using a microscope camera. The scissor displacement was measured from the captured images by counting pixels; the $1.16 \mu \mathrm{m}$ pixelization dominates the measurement error.

The measured peak-to-peak displacement is plotted in Figure 7. The markers represent data points, and the error bars reflect a $+/-$ one pixel measurement error. The solid lines are the displacement predicted analytically for a tactel with an ideal, pinned-hinge scissor. For the purposes of this calculation, the flexural hinges are approximated as pinned hinges located on the neutral plane of the flexural hinge directly adjacent to the anchor support and the central pin. For the device with an SU-8 scissor, the displacement ranges from a minimum of of $3.8 \mu \mathrm{m}$ measured at $40 \mathrm{~V}$ to a maximum of $10.7 \mu \mathrm{m}$ measured at $170 \mathrm{~V}$. For the device with a 3D printed scissor, the displacement ranges from $2.7 \mu \mathrm{m}$ to $8.9 \mu \mathrm{m}$ over that voltage range. In all cases, the increase in displacement with applied voltage is similar to the linear trend that is predicted using the ideal pinned hinge model. The FEA model slightly under-predicts the measured displacement at $95 \mathrm{~V}(2 \mu \mathrm{m}$ peak predicted as compared with $\sim 3 \mu \mathrm{m}$ peak determined from the measured $\sim 6 \mu \mathrm{m}$ peak-to-peak displacement). The larger displacement of the SU-8 scissor for the same voltage reflects the SU-8 scissor's thinner hinge and narrower width, both of which reduce its mechanical stiffness under horizontal actuation.

To measure the amplitude of the force that the tactels apply when they vibrate in contact with a rigid surface, the individual tactels were mounted on a second testing stage that is mounted to the grips of a mechanical tester (Instron 5943). The tensile tester was preloaded to apply a force of $40 \mathrm{mN}$ to the sensing pin when zero voltage was applied. A square wave voltage with a frequency of $10 \mathrm{~Hz}$ was applied to the tactel, and its peak amplitude was increased from $40 \mathrm{~V}$ to $170 \mathrm{~V}$ in increments of $10 \mathrm{~V}$. The resulting forces were measured by the mechanical tester's load cell. The raw data were post-processed in MATLAB to eliminate signals at frequencies other than $10 \mathrm{~Hz}$ (noise and drift).

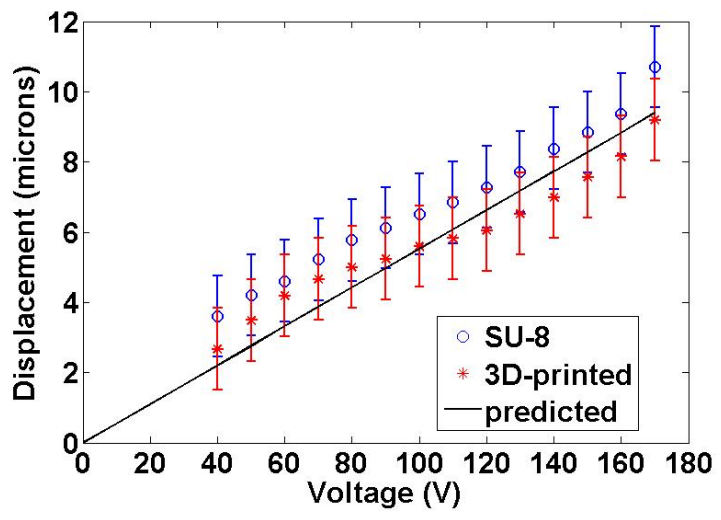

Figure 7: Plot of tactel displacement vs. applied voltage measured with $S U-8$ and $3 D$-printed scissors and predicted for ideal scissors. 
Figure 8 plots the measured peak-to-peak amplitude of the tactel's oscillating force. The markers represent the measured forces, and the connecting lines are included as guides to the eye. Each force measurement is repeated over 15 to 20 voltage cycles, resulting in 15 to 20 nominally identical force measurements. The error bars represent the maximum variation that was observed between nominally identical repeated measurements. Whereas the measured displacements were similar for the SU-8 and 3-D printed devices and matched the models well in all cases, the measured forces differ widely between the designs. The forces from the tactel with the $3 \mathrm{D}$ printed scissor have larger values, increasing from $6.9 \mathrm{mN}$ at $40 \mathrm{~V}$ to $48.7 \mathrm{mN}$ at $170 \mathrm{~V}$. The forces from the tactel with the SU-8 scissor are smaller, ranging from $2.3 \mathrm{mN}$ to $14.7 \mathrm{mN}$. The lower forces from the tactel with the SU-8 scissor primarily reflect the SU-8 scissor's lower stiffness due to differences in geometry (hinge thickness and width); their Young's modulus values are similar.

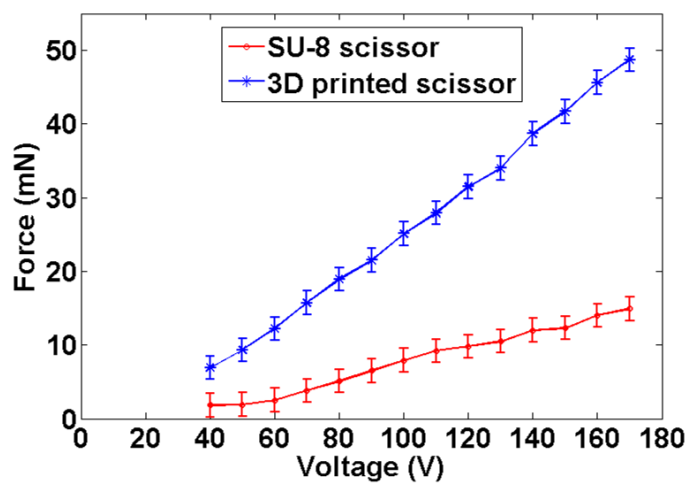

Figure 8: Measured tactel force vs. peak amplitude of the applied voltage. Markers represent data; lines are guides to the eye.

Individual tactels with SU-8 and 3D-printed scissors were tested by sighted volunteers to determine the minimum voltages at which vibration can be sensed and is comfortable. Each measurement was repeated at $20 \mathrm{~Hz}, 100 \mathrm{~Hz}$, and $250 \mathrm{~Hz}$. Although the small number of testers to date precludes a statistically significant analysis, several initial trends emerge. At all three frequencies, the level of comfortable vibration is typically $\sim 2-4 \mu \mathrm{m}$. For 3D-printed scissors, the comfortable level of force is typically around $\sim 8-10 \mathrm{mN}$, whereas SU-8 scissors enable comfortable detection at the $\sim 2 \mathrm{mN}$ scale. The difference in necessary force for SU-8 and 3D printed scissors indicates either that sensing is dominated by displacement rather than by scissor deformability in this design range, or that the details of the pin's shape (e.g. SU-8's square corners as compared with the 3D printed pin's rounded top) are critical determinants of the force required for sensing. The minimum detectable levels are lower than the values for comfortable usage. Since the minimum detectable voltages (typically $\sim 10-30 \mathrm{~V}$ ) lie below $40 \mathrm{~V}$, the corresponding forces and displacements cannot be determined from Figures 7 and 8. Higher frequencies typically permit sensing of smaller forces and displacements, in agreement with the literature [11].

Sensing of the tactile arrays was less robust and required higher voltages. The difficulty of sensing vibrations in the arrays is attributed to the thin foam interlayer included in the array package. Although this layer successfully minimizes parasitic vibrations to neighboring pins, it has a detrimental effect on the sensed vibrational amplitude on the target pin. Future work will focus in part on minimizing vibrational damping at the target pin.

\section{CONCLUSION}

Tactile elements based on the actuator plus amplifier design are shown to be effective at the milliscale. Their measured performance agrees with the models, with maximum deflections of greater than $10 \mu \mathrm{m}$ and maximum forces above $45 \mathrm{mN}$ that place the devices well above the sensing threshold. An analytical model based on ideal pinned hinges is shown to be useful for predicting the behavior of tactels with flexural hinges, especially when coupled with FEA to predict hinge failure. The analytical model validation provides support for further downscaling of the tactile elements to the $1-2 \mathrm{~mm}^{2}$ scale, since the models also predict successful functioning at this scale. At the MEMS scale, the display's resolution would correspond to up to 100 tactels $/ \mathrm{cm}^{2}$. The measured performance confirms sensing thresholds of less than $4 \mu \mathrm{m}$ and $2 \mathrm{mN}$ for the most effective tactile devices.

\section{ACKNOWLEDGMENTS}

The authors thank Patrick Murphy and Constantinos Mavroidis for assistance with the 3D printing, which was done in Northeastern's Biomedical Mechatronics Laboratory. The research was funded by the Andrea Bocelli Foundation. Travel support has been generously provided by the Transducer Research Foundation.

\section{REFERENCES}

[1] M. Nakajima, K. Suzuki, H.R. Su, Y. Tani, and A. Morita, "Braille Cell", U.S. Patent 5226817, 1991.

[2] Y. Tani, Y. Shiraishi, H.R. Su, A. Morita, and K. Suzuki, "Tactile reading device", US Patent 5449292, 1993.

[3] J.C. Bliss, M.N. Katcher, C.H. Rogers, and R.P. Shepard, "Optical-to-tactile image conversion for the blind", IEEE Transactions of Man-Machine Systems, 11, 1, pp. 58 - 65, (1970).

[4] Y. Bar-Cohen, "Refreshable Braille displays using EAP actuators" SPIE 7642 Electroactive Polymer Actuators and Devices, International Society for Optics and Photonics, San Diego, CA, (2010), pp. 764206 - 76420.

[5] J. Pasquero and V. Hayward, "STReSS: A Practical Tactile Display System with One Millimeter Spatial Resolution and $700 \mathrm{~Hz}$ Refresh Rate", Proc. of Eurohaptics 2003, Dublin, Ireland (2003), pp. 94 - 110.

[6] Metec AG, http://web.metec-ag.de/cell\%20d2.html

[7] N. Kitamura, J. Chim, N. Miki, "Micro-needle electrode array for electro tactile display", Technical Digest of the 2013 Transducers, Barcelona, Spain, Transducer Research Foundation, (2013), pp. 106 - 107.

[8] N. Torras et al., "Nematic opto-mechanical actuators for the fabrication of refreshable tactile systems", Technical Digest of the 2013 Transducers, Barcelona, Spain, Transducer Research Foundation, (2013), pp. 1691 - 1694.

[9] Ninomiya, T., Y. Okayama, Y. Matsumoto, X. Arouette, K. Osawa, and N. Miki. "MEMS-based hydraulic displacement amplification mechanism with completely encapsulated liquid." Sensors and Actuators A: Physical, 166, 2: pp. 277 282. (2001).

[10] J. Downar, A.P. Crawley, D.J. Mikulis, and K.D. Davis, “A multimodal cortical network for the detection of changes in the sensory environment", Nature Neuroscience, 3, pp. 277 283, (2000).

[11] E. Kandel, J. Schwartz and T. Jessell's, "Principles of Neural Science (Fourth Edition)," McGraw-Hill, New York, 2000.

\section{CONTACT}

*X.Xie, tel: +1-617-373-2048; x.xie@neu.edu 